\title{
Adiabatic Theory of Electron Detachment from Negative Ions in Two-Color Laser Field
}

\author{
M. Yu. Kuchiev and V. N. Ostrovsky甘 \\ School of Physics, University of New South Wales, Sydney 2052, Australia
}

\begin{abstract}
Negative ion detachment in bichromatic laser field is considered within the adiabatic theory. The latter represents a recent modification of the famous Keldysh model for multiphoton ionization [L. V. Keldysh, Zh. Eksp. Teor. Fiz. 47, 1945 (1964) [Sov. Phys.-JETP 20, 1307 (1965)]] which makes it quantitatively reliable. We calculate angular differential detachment rates, partial rates for particular ATD (Above Threshold Detachment) channels and total detachment rates for $\mathrm{H}^{-}$ion in a bichromatic field with $1: 3$ frequency ratio and various phase differences. Reliability of the present, extremely simple approach is testified by comparison with much more elaborate earlier calculations.
\end{abstract}

PACS numbers: 32.80.Rm, 32.80.Fb

Typeset using REVTEX 


\section{INTRODUCTION}

Interest to the photoionization of atoms in a bichromatic laser field both in theory (see, for instance, Ref. [1]- [13]) and in experiment [1] [14]- [16] seems to stem first of all from the effect of the phase control, i.e. dependence of the observables on the difference of field phases $\varphi$.

The calculations have been carried out previously for ionization of the hydrogen atom in two laser fields with a frequency ratio $1: 2$ [4], $1: 3$ [5] and $2: 3$ [7]. Potvliege and Smith [6] presented results for various frequency ratios and initial states. Different schemes have been employed, but all of them imply numerically intensive work.

For the multiphoton electron detachment from negative ions some analytical treatment exists [8] [9] which aims to investigate qualitative features of the process, mostly in the case when one or both fields are weak. The presence of large number of parameters in the problem sometimes makes results of analytical studies not directly transparent. Quantitative reliability of these approaches has never been assessed. This situation looks particularly unsatisfactory since the multiphoton electron detachment from negative ions presents unique situation when quantitative results can be obtained by analytical method in a broad range of parameters characteristic to the problem. Indeed, it has been demonstrated recently by Gribakin and Kuchiev [17] [18] that proper application of the well-known Keldysh [19] model to multiphoton detachment 20] provides an extremely simple scheme that gives very reliable results for the total rates as well as for ATD (Above Threshold Detachment) spectrum and ATD angular distributions. This adiabatic approximation ensures an accuracy which is comparable with that of the most elaborate numerical developments and works unexpectedly well even outside its formal applicability range, i.e. even for small number $n$ of photons absorbed. The evidences of good performance of the Keldysh model for the total rates were presented also in the earlier paper [25].

Recently the adiabatic approach was extended by the present authors [26] to the case of bichromatic field. The practical applications were carried out for the case of frequency 
ratio $1: 2$ when in addition to the phase effects another unusual phenomenon exists, namely the polar asymmetry of the angular distribution of photoelectrons. Unfortunately no other quantitative data for photodetachment in this case is available which makes comparison impossible.

The main objective of the present study is to assess quantitatively an accuracy of the adiabatic scheme by comparison with the previous calculations carried out by Telnov et al [11] in case of $1: 3$ frequency ratio. For this ratio the polar asymmetry is absent, but the phase effects persist. The calculations by Telnov et al [11] are based on sufficiently sophisticated numerical scheme providing a useful benchmark. We present (Sec. II) complete comparison of the results by considering angular differential detachment rates, heights of ATD peaks and total detachment rates. It should be emphasized that the angular differential rates are most sensitive to the formulation of the model representing an ultimate test for the theory, as discussed in Sec.III. We draw also some general conclusion on the relation between the adiabatic approach and the numerical calculations within the one-electron approximation.

\section{RESULTS}

The adiabatic theory of two-color detachment was outlined in our previous paper 26] where the reader can find all the details of calculation. Here we only write down the expression for the electric field strength in the bichromatic laser field with $1: 3$ frequency ratio in order to specify the definition of the field phase difference $\varphi$

$$
\vec{F}(t)=\vec{F}_{1} \cos \omega t+\vec{F}_{2} \cos (3 \omega t+\varphi)
$$

$\vec{F}_{1}, \vec{F}_{2}$ are the amplitude vectors for the fundamental frequency $\omega$ and its third harmonics respectively. Below we consider, just as in Ref. [11], the case when both fundamental field and its third harmonics are linear polarized with $\vec{F}_{1} \| \vec{F}_{2}$. Then the differential photoionization rate depends only on the single angle $\theta$ between the photoelectron translational momentum

$\vec{p}$ and the vectors $\vec{F}_{1} \| \vec{F}_{2}$. Atomic units are used throughout the paper unless stated otherwise. 
Our calculations for $\mathrm{H}^{-}$detachment are carried out for the parameters of $\mathrm{H}^{-}$as before 26] $(\kappa=0.2354, A=0.75)$. We choose two sets of field intensities $I_{1}$ and $I_{2}$ for the fundamental frequency $\omega=0.0043$ (that of $\mathrm{CO}_{2}$ laser) and its third harmonics, same as in the paper by Telnov et al [11], namely (i) $I_{1}=10^{10} \mathrm{~W} / \mathrm{cm}^{2}, I_{2}=10^{9} \mathrm{~W} / \mathrm{cm}^{2}$ and (ii) $I_{1}=10^{10} \mathrm{~W} / \mathrm{cm}^{2}, I_{2}=10^{8} \mathrm{~W} / \mathrm{cm}^{2}$.

In case of the frequencies ratio $1: 3$ considered here the field (1) does not possess polar asymmetry (i.e. asymmetry under inversion of the $z$ axis directed along $\vec{F}_{1} \| \vec{F}_{2}$ ). Therefore the differential detachment rate does not change under the transformation $\theta \Rightarrow \pi-\theta$. This allows us to show plots only for $\frac{1}{2} \pi \geq \theta \geq 0$ domain.

Figs. 1 16 show the differential detachment rate as a function of the angle $\theta$ for three lowest (open) ATD channels and for two sets of field intensities. The system Hamiltonian is a $2 \pi$-periodic function of the phase parameter $\varphi$. We show our results for $\varphi=0, \varphi= \pm \frac{1}{2}$ and $\varphi=\pi$. The transformation $\varphi \Rightarrow \pi-\varphi$ leaves the Hamiltonian invariant only if $t$ is replaced by $-t$. As stressed in Refs. [2], the problem is invariant under the time inversion operation provided the final-state electron interaction with the atomic core is neglected. This is the case in the present model. Therefore our differential ionization rates are the same for $\varphi$ and $-\varphi$. The calculations by Telnov et al [11] do take into account the final state electron-core interaction. Therefore they show some difference between the angular differential rates for $\varphi$ and $-\varphi$. However, it proves to be quite small for low ATD channels as seen from the plots.

The importance of the interaction between the emitted electron and the core has been first pointed out by one of the present authors [27]. In this paper several phenomena has been predicted for which this interaction plays crucial role. The related mechanism was named "atomic antenna". In the recent literature the final state interaction is usually referred to as rescattering. In our problem the rescattering effects are enhanced for high ATD channels as discussed below.

The results of our extremely simple theory are compared in Figs. 1-6 with the previous numerical calculations by Telnov et al [11] which are rather involving. Being carried out in the one-electron approximation, they employ an accurate model for the effective one-electron 
potential in $\mathrm{H}^{-}$[28], complex-scaling generalized pseudospectral technique [29] to discretize and facilitate the solution of the time-independent non-Hermitian Floquet Hamiltonian for complex quasienergies and eigenfunctions, and calculation of the electron energy and angular distributions by the reverse complex-scaling method [30]. As a lucid illustration of simplicity of the present approach it is worthwhile to stress that it does not rely on any particular form of an effective one-electron potential albeit employs only two parameters $\kappa$ and $A$ governing the asymptotic behavior of the initial bound state wave function.

From Figs. 116 one can see that the adiabatic approximation ensures good quantitative agreement with calculations by Telnov et al [11. In particular, positions of maxima and minima in the angular photoelectron distribution are well reproduced. This demonstrates that the adiabatic approach correctly describes the nature of the structure as due to interference between the electron waves emitted at various (complex-valued) moments of time. Indeed, within the adiabatic theory [26] the ionization amplitude is expressed as a sum of a number of interfering contributions. Mathematically they come from different saddle points in the approximate evaluation of the integral over time that emerges in the Keldysh [19] model. Physically they correspond to the coherent emission of photoelectron at different moments of time. For our particular frequency ratio $1: 3$ the sum contains 6 interfering contributions as compared with 4 term for $1: 2$ frequency ratio [26] and 2 terms for onecolor detachment [17] [18]. Generally this suggests that in the former case more complicated angular patterns emerge. Probably one can find here a correlation with an alternative interpretation in the multiphoton absorption framework. The latter argues [7] [11] that the angular distribution structure in $1: 3$ case is more complicated than for $1: 2$ ratio since all the pathways leading to a continuum state with the same energy interfere in the $1: 3$ case whereas a considerable pattern of non-interfering pathways exists for the the $1: 2$ case due to parity or energy restrictions (each pathway is characterized by the number of photons of different colors absorbed successively).

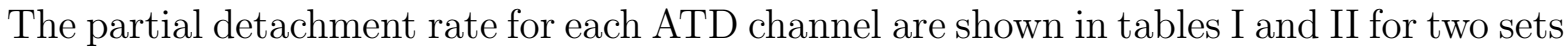
of field intensities. The agreement is good for low ATD channels; note that the rescattering 
effects which generate dependence on the sign of $\varphi$ are manifested in the partial rates even less than in the angular distributions shown in Figs. 1-6. For higher ATD channels with low rates the difference between the present results and those of Telnov et al [11] becomes more pronounced. This behavior could be interpreted as increasing importance of rescattering for high ATD peaks. The manifestations of this effect were observed recently in experiment [31] and are currently vividly discussed in the literature [27] [31] [32] [33].

\section{CONCLUSION}

As a summary, the adiabatic approach provides quantitatively reliable tool for investigating two-color photodetachment of negative ions. In particular, the interference structure in the photoelectron angular distributions as well as the phase effects are correctly described. Since generally the interference phenomena are known to be most sensitive to the details of theoretical description, one can conclude that the present theory had successfully passed the stringent test.

The Keldysh scheme [19] is known to be gauge-noninvariant. Importantly, the calculations within the adiabatic approach [17] [18] [26] employ the dipole-length gauge for the laser field thus stressing contribution of the long-range asymptote of the initial bound state wave function. The use of the length gauge together with the adiabatic approach (i.e. integration over time by the saddle point method, see Refs. [17] [18] [26] and discussion in Sec.[1]) render self-consistent character to the theoretical scheme. Indeed, the exact evaluation of the integrals does not add to the accuracy of the result as compared with the use of the saddle point method. This is because in the former case the integral absorbs the contributions from the wave function outside its asymptotic domain, where in fact it is known with much lower accuracy (being, in particular, influenced by the effects beyond the single active electron approximation).

The method is straightforwardly applicable to the negative ions with the outer electron

having non-zero orbital momentum, such as halogen ions, which could be easier accessible 
for the experimental studies (for the one-color detachment such applications could be found in Ref. [17]). Technically the calculations within the adiabatic approach are extremely simple reducing to finding the roots of polynomial and substituting them into an analytical expression [26] (the related Mathematica [34] program takes only few lines). It should be recognized that the single active electron approximation itself introduces some intrinsic error. It seems that often this error could be comparable with the difference between the result of numerical one-electron calculations and these of the adiabatic approximation. Uncertainty of the one-electron approach in principle could be removed within the two-electron approach which however consumes much more efforts. The two-electron calculations which has been carried out recently show that the one-electron approximation is generally sufficient unless one is particularly interested in the subtle resonance effects [25] [35] [36] (the calculations beyond one-electron approximation are currently possible only for small number of absorbed photons). For high ATD channels with low intensities the adiabatic approximation becomes less reliable due to increasing role of rescattering effects neglected in the present form of the approximation. It seems however that relatively simple modifications of the adiabatic approximation could be carried to include rescattering effects.

Reliability of the results obtained above for the simple one-electron problem with rescattering neglected is highly important in perspective, since they are to be included as a constituent part in the treatment of much more sophisticated one-electron and many-electron problems governed by the antenna mechanism [27] [37] 38.

\section{ACKNOWLEDGMENTS}

We appreciate fruitful discussions with G. F. Gribakin. We are grateful to the referee of the present paper for attracting our attention to Ref. [25]. The support from the Australian Research Council is thankfully acknowledged. V. N. O. acknowledges a hospitality of the stuff of the School of Physics of UNSW where this work has been carried out. 


\section{REFERENCES}

* Permanent address: Institute of Physics, The University of St Petersburg, 198904 St Petersburg, Russia; E-mail: Valentin.Ostrovsky@pobox.spbu.ru

[1] N. B. Baranova, B. Ya. Zel'dovich, A. N. Chudinov, and A. A. Shul'ginov, Zh. Eksp. Teor. Fiz. 98, 1857 (1990) [Sov. Phys.-JETP 71, 1043 (1990)]; N. B. Baranova, I. M. Beterov, B. Ya. Zel'dovich, I. I. Ryabtsev, A. N. Chudinov, and A. A. Shul'ginov, Pis'ma Zh. Eksp. Teor. Fiz. 55, 431 (1992) [JETP Letters 55, 439 (1992)].

[2] N. B. Baranova and B. Ya. Zel'dovich, J. Opt. Soc. Am. B 8, 27 (1991); D. Z. Anderson, N. B. Baranova, K. Green, and Ya. B. Zel'dovich, Zh. Eksp. Teor. Fiz. 102, 397 (1992) [Sov. Phys.-JETP 75, 210 (1992)].

[3] A. Szöke, K. C. Kulander, and J. N. Bardsley, J. Phys. B 24, 3165 (1991).

[4] K. J. Schafer and K. C. Kulander, Phys. Rev. A 45, 8026 (1992).

[5] R. M. Potvliege and P. H. G. Smith, J. Phys. B 24, L641 (1991).

[6] R. M. Potvliege and P. H. G. Smith, J. Phys. B 25, 2501 (1992).

[7] R. M. Potvliege and P. H. G. Smith, Phys. Rev. A 49, 3110 (1994).

[8] N. B. Baranova, H. R. Reiss, and B. Ya. Zel'dovich, Phys. Rev. A 48, 1497 (1993).

[9] V. A. Pazdzersky and V. A. Yurovsky, Phys. Rev. A 51, 632 (1994); Laser Physics 5, 1141 (1995); V. A. Pazdzersky and V. I. Usachenko, J. Phys. B 30, 3387 (1997); Laser Physics 5, 1137 (1995).

[10] M. Protopapas, P. L. Knight, and K. Burnett, Phys. Rev. A 49, 1945 (1994).

[11] D. A. Telnov, J. Wang, and S. I. Chu, Phys. Rev. A 51, 4797 (1995).

[12] V. Véniard, R. Täieb, and A. Maquet, Phys. Rev. Lett. 74, 4161 (1995).

[13] M. Fifirig, A. Cionga and V. Florescu, J. Phys. B 30, 2599 (1997). 
[14] H. G. Muller, P. H. Bucksbaum, D. W. Schumacher, and A. Zavryiev, J. Phys. B 23, $2761(1990)$.

[15] Ce Chen, Y.-Y. Yin, and D. S. Elliott, Phys. Rev. Lett. 64, 507 (1990); Ce Chen and D. S. Elliott, Phys. Rev. Lett. 65, 1737 (1990); Y.-Y. Yin, Ce Chen, D. S. Elliott, and A. V. Smith, Phys. Rev. Lett. 69, 2353 (1992).

[16] D. W. Schumacher, F. Weihe, H. G. Muller, and P. H. Bucksbaum, Phys. Rev. Lett. 73, 1344 (1994).

[17] G. F. Gribakin and M. Yu. Kuchiev, Phys. Rev. A 55, 3760 (1997).

[18] G. F. Gribakin and M. Yu. Kuchiev, J. Phys. B 30, L657 (1997); 31, 3087 (1998).

[19] L. V. Keldysh, Zh. Eksp. Teor. Fiz. 47, 1945 (1964) [Sov. Phys.-JETP 20, 1307 (1965)].

[20] Subsequent development of the Keldysh model was due to Perelomov et al [21], Faisal [22] and Reiss [23]; Perelomov and Popov [24] were the first to consider multicolour process within this framework (in terms of influence of higher harmonics on ionization probabilities).

[21] A. M. Perelomov and V. S. Popov, and M. V. Terent'ev, Zh. Eksp. Teor. Fiz. 50, 1393 (1966) [Sov. Phys.-JETP 23, 924 (1966)].

[22] F. H. M. Faisal, J. Phys. B 6, L89 (1973).

[23] H. R. Reiss, Phys. Rev. A 22, 1786 (1980).

[24] A. M. Perelomov and V. S. Popov, Zh. Eksp. Teor. Fiz. 52, 514 (1967) [Sov. Phys.-JETP 25, $336(1967)]$.

[25] M. Dörr, R. M. Potvliege, D. Proulx, and R. Shakeshaft, Phys. Rev. A 42, 4138 (1990).

[26] M. Yu. Kuchiev and V. N. Ostrovsky, J. Phys. B 31, 2525 (1998).

[27] M. Yu. Kuchiev, Pis'ma Zh. Eksp. Teor. Fiz. 45, 404 (1987) [JETP Letters 45, 319 
(1987)].

[28] C. Laughlin and S. I. Chu, Phys. Rev. A 48, 4654 (1993).

[29] J. Wang, S. I. Chu, and C. Laughlin, Phys. Rev. A 50, 3208 (1994); G. Yao and S. I. Chu, Chem. Phys. Lett. 204, 381 (1993).

[30] D. A. Telnov and S. I. Chu, Phys. Rev. A 50, 4099 (1994).

[31] K. J. Schafer, B. Yang, L. F. DiMauro, and K. C. Kulander, Phys. Rev. Lett. 70, 1599 (1993); B. Yang, K. J. Schafer, B. Walker, K. C. Kulander, P. Agostini, and L. F. DiMauro. Phys. Rev. Lett. 71, 3770 (1993); G. G. Paulus, W. Nicklich, Huale Xu, P. Lambropoulos, and H. Walther, Phys. Rev. Lett. 72, 2851 (1994); G. G. Paulus, W. Nicklich, F. Zacher, P. Lambropoulos, and H. Walther, J. Phys. B 29, L249 (1996); G. G. Paulus, W. Nicklich, and H. Walther, Europhys. Lett. 27, 267 (1994); B. Walker, B. Sheehy, K. C. Kulander, and L. F. DiMauro, Phys. Rev. Lett. 77, 5031 (1996).

[32] X. Mu, Phys. Rev. A 42, 2944 (1990); X. Mu, J. Ruscheinsky, and B. Crasemann, Phys.Rev. A 42, 2949 (1990); W. Becker, A. Lohr and M. Kleber, J. Phys. B 27, L325 (1994); 28, 1931 (1995); G. G. Paulus, W. Becker, W. Nicklich, and H. Walther, J. Phys. B 27, L703 (1994); G. G. Paulus, W. Becker, and H. Walther, Phys. Rev. A 52, 4043 (1995); M. Lewenstein, K. C. Kulander, K. J. Schafer, and P. H. Bucksbaum, Phys. Rev. A 51, 1495 (1995); A. Lohr, M. Kleber, R. Kopold, and W. Becker, Phys. Rev. A 55, R4003 (1997); J. Z. Kamińsky, A. Jaroń, and F. Ehlotzky, Phys. Rev. A 53, 1756 (1996); J. Z. Kamińsky and F. Ehlotzky, J. Phys. B 30, 69, 5729 (1997); E. Cormier and P. Lambropoulos, J. Phys. B 30, 77 (1997); S. Dionissopoulou, Th. Mercouris, A. Lyras, and C. A. Nicolaides, Phys. Rev. A 55, 4397 (1997); D. B. Milosević and F. Ehlotzky, Phys. Rev. A 57, 5002 (1998).

[33] D. F. Zaretskii and E. A. Nersesov, Zh. Eksp. Teor. Fiz. 103, 1192 (1993) [JETP 76, 583 (1993)]. 
[34] S. Wolfram, Mathematica: A System for Doing Mathematics by Computer, 2nd ed. (Addison-Wesley Publishing Co., Palo Alto, 1991).

[35] L. A. A. Nikolopoulos and P. Lambropoulos, Phys. Rev. A 56, 3106 (1997).

[36] I. Sánchez, H. Bachau, and F. Martin, J. Phys. B 30, 2417 (1997).

[37] M. Yu. Kuchiev, J. Phys. B 28, 5093 (1995).

[38] M. Yu. Kuchiev. Phys. Lett. A 212, 77 (1996). 


\section{TABLES}

TABLE I. Partial rates for the $\mathrm{H}^{-}$detachment by the laser wave with the frequency $\omega=0.0043$ and its third harmonics with the intensities respectively $I_{1}=10^{10} \mathrm{~W} / \mathrm{cm}^{2}$ and $I_{2}=10^{9} \mathrm{~W} / \mathrm{cm}^{2}$. The number of absorbed photons $n$ refers to the fundamental frequency. In each block the upper figure gives present result and the lower one the result obtained by Telnov et al [11]. The number in square brackets indicate the power of 10 .

\begin{tabular}{|c|c|c|c|c|c|c|}
\hline \multirow{2}{*}{$n$} & \multirow{2}{*}{$\begin{array}{l}\text { One-colour } \\
\text { fundamental }\end{array}$} & \multicolumn{4}{|c|}{ Two-colour } & \multirow{2}{*}{$\begin{array}{l}\text { One-colour } \\
\text { harmonic }\end{array}$} \\
\hline & & $\varphi=0$ & $\varphi=\pi$ & $\varphi=\frac{1}{2} \pi$ & $\varphi=-\frac{1}{2} \pi$ & \\
\hline \multirow[t]{2}{*}{8} & $0.67[-9]$ & $0.47[-7]$ & $0.54[-8]$ & $0.22[-7]$ & $0.22[-7]$ & \\
\hline & $0.72[-9]$ & $0.42[-7]$ & $0.58[-8]$ & $0.20[-7]$ & $0.21[-7]$ & \\
\hline \multirow[t]{2}{*}{9} & $0.20[-9]$ & $0.11[-7]$ & $0.23[-8]$ & $0.80[-8]$ & $0.80[-8]$ & $0.46[-10]$ \\
\hline & $0.20[-9]$ & $0.10[-7]$ & $0.23[-8]$ & $0.71[-8]$ & $0.73[-8]$ & $0.30[-10]$ \\
\hline \multirow[t]{2}{*}{10} & $0.41[-10]$ & $0.27[-8]$ & $0.34[-8]$ & $0.39[-8]$ & $0.39[-8]$ & \\
\hline & $0.39[-10]$ & $0.26[-8]$ & $0.27[-8]$ & $0.35[-8]$ & $0.30[-8]$ & \\
\hline \multirow[t]{2}{*}{11} & $0.50[-11]$ & $0.65[-9]$ & $0.23[-8]$ & $0.17[-8]$ & $0.17[-8]$ & \\
\hline & $0.40[-11]$ & $0.72[-9]$ & $0.16[-8]$ & $0.15[-8]$ & $0.10[-8]$ & \\
\hline \multirow[t]{2}{*}{12} & $0.74[-12]$ & $0.16[-9]$ & $0.10[-8]$ & $0.62[-9]$ & $0.62[-9]$ & $0.66[-13]$ \\
\hline & $0.71[-12]$ & $0.20[-9]$ & $0.71[-9]$ & $0.58[-9]$ & $0.30[-9]$ & $0.86[-13]$ \\
\hline
\end{tabular}




\begin{tabular}{|c|c|c|c|c|c|c|}
\hline 13 & $0.21[-12]$ & $0.38[-10]$ & $0.36[-9]$ & $0.20[-9]$ & $0.20[-9]$ & \\
\hline & $0.33[-12]$ & $0.53[-10]$ & $0.27[-9]$ & $0.20[-9]$ & $0.85[-10]$ & \\
\hline \multirow[t]{2}{*}{14} & $0.64[-13]$ & $0.88[-11]$ & $0.11[-9]$ & $0.58[-10]$ & $0.58[-10]$ & \\
\hline & $0.14[-12]$ & $0.14[-10]$ & $0.97[-10]$ & $0.69[-10]$ & $0.32[-10]$ & \\
\hline \multirow[t]{2}{*}{15} & $0.16[-13]$ & $0.20[-11]$ & $0.32[-10]$ & $0.16[-10]$ & $0.16[-10]$ & $0.16[-15]$ \\
\hline & $0.47[-13]$ & $0.32[-11]$ & $0.33[-10]$ & $0.23[-10]$ & $0.17[-10]$ & $0.31[-15]$ \\
\hline \multirow[t]{2}{*}{16} & $0.36[-14]$ & $0.52[-12]$ & $0.82[-11]$ & $0.44[-11]$ & $0.44[-11]$ & \\
\hline & $0.14[-13]$ & $0.72[-12]$ & $0.12[-10]$ & $0.78[-11]$ & $0.10[-10]$ & \\
\hline \multirow[t]{2}{*}{17} & $0.68[-15]$ & $0.15[-12]$ & $0.21[-11]$ & $0.12[-11]$ & $0.12[-12]$ & \\
\hline & $0.35[-14]$ & $0.22[-12]$ & $0.43[-11]$ & $0.27[-11]$ & $0.60[-11]$ & \\
\hline \multirow[t]{2}{*}{ Total } & $0.92[-9]$ & $0.62[-7]$ & $0.15[-7]$ & $0.36[-7]$ & $0.36[-7]$ & $0.46[-7]$ \\
\hline & $0.96[-9]$ & $0.56[-7]$ & $0.14[-7]$ & $0.33[-7]$ & $0.33[-7]$ & $0.30[-7]$ \\
\hline
\end{tabular}


TABLE II. Same as in table 1 , but for the intensities $I_{1}=10^{10} \mathrm{~W} / \mathrm{cm}^{2}$ and $I_{2}=10^{8} \mathrm{~W} / \mathrm{cm}^{2}$.

\begin{tabular}{|c|c|c|c|c|c|c|}
\hline \multirow{2}{*}{$n$} & \multirow{2}{*}{$\begin{array}{l}\text { One-colour } \\
\text { fundamental }\end{array}$} & \multicolumn{4}{|c|}{ Two-colour } & \multirow{2}{*}{$\begin{array}{l}\text { One-colour } \\
\text { harmonic }\end{array}$} \\
\hline & & $\varphi=0$ & $\varphi=\pi$ & $\varphi=\frac{1}{2} \pi$ & $\varphi=-\frac{1}{2} \pi$ & \\
\hline \multirow[t]{2}{*}{8} & $0.67[-9]$ & $0.54[-8]$ & $0.41[-9]$ & $0.25[-8]$ & $0.25[-8]$ & \\
\hline & $0.72[-9]$ & $0.53[-8]$ & $0.36[-9]$ & $0.24[-8]$ & $0.25[-8]$ & \\
\hline \multirow[t]{2}{*}{9} & $0.20[-9]$ & $0.92[-9]$ & $0.16[-9]$ & $0.69[-9]$ & $0.69[-9]$ & $0.46[-13]$ \\
\hline & $0.20[-9]$ & $0.93[-9]$ & $0.18[-9]$ & $0.68[-9]$ & $0.68[-9]$ & $0.30[-13]$ \\
\hline \multirow[t]{2}{*}{10} & $0.41[-10]$ & $0.23[-9]$ & $0.82[-10]$ & $0.25[-9]$ & $0.25[-9]$ & \\
\hline & $0.39[-10]$ & $0.25[-9]$ & $0.82[-10]$ & $0.25[-9]$ & $0.22[-9]$ & \\
\hline \multirow[t]{2}{*}{11} & $0.50[-11]$ & $0.54[-10]$ & $0.74[-10]$ & $0.88[-10]$ & $0.88[-10]$ & \\
\hline & $0.40[-11]$ & $0.59[-10]$ & $0.65[-10]$ & $0.88[-10]$ & $0.66[-10]$ & \\
\hline \multirow[t]{2}{*}{12} & $0.74[-12]$ & $0.11[-10]$ & $0.38[-10]$ & $0.28[-10]$ & $0.28[-10]$ & $0.66[-17]$ \\
\hline & $0.71[-12]$ & $0.14[-10]$ & $0.33[-10]$ & $0.28[-10]$ & $0.22[-10]$ & $0.98[-17]$ \\
\hline \multirow[t]{2}{*}{13} & $0.21[-12]$ & $0.21[-11]$ & $0.13[-10]$ & $0.79[-11]$ & $0.79[-11]$ & \\
\hline & $0.33[-12]$ & $0.45[-11]$ & $0.13[-10]$ & $0.82[-11]$ & $0.94[-11]$ & \\
\hline \multirow[t]{2}{*}{14} & $0.64[-13]$ & $0.39[-12]$ & $0.39[-11]$ & $0.20[-11]$ & $0.20[-11]$ & \\
\hline & $0.14[-12]$ & $0.21[-11]$ & $0.47[-11]$ & $0.24[-11]$ & $0.49[-11]$ & \\
\hline
\end{tabular}




\begin{tabular}{|c|c|c|c|c|c|c|}
\hline \multirow[t]{2}{*}{15} & $0.16[-13]$ & $0.66[-13]$ & $0.96[-12]$ & $0.46[-12]$ & $0.46[-12]$ & $0.16[-21]$ \\
\hline & $0.47[-13]$ & $0.11[-11]$ & $0.17[-11]$ & $0.70[-12]$ & $0.25[-11]$ & \\
\hline \multirow[t]{2}{*}{16} & $0.36[-14]$ & $0.11[-13]$ & $0.21[-12]$ & $0.99[-13]$ & $0.99[-13]$ & \\
\hline & $0.14[-13]$ & $0.52[-12]$ & $0.60[-12]$ & $0.21[-12]$ & $0.12[-11]$ & \\
\hline \multirow[t]{2}{*}{17} & $0.68[-15]$ & $0.17[-14]$ & $0.42[-13]$ & $0.21[-13]$ & $0.21[-13]$ & \\
\hline & $0.35[-14]$ & $0.22[-12]$ & $0.22[-12]$ & $0.64[-13]$ & $0.50[-12]$ & \\
\hline \multirow[t]{2}{*}{ Total } & $0.92[-9]$ & $0.66[-8]$ & $0.79[-9]$ & $0.36[-8]$ & $0.36[-8]$ & $0.46[-13]$ \\
\hline & $0.96[-9]$ & $0.66[-8]$ & $0.74[-9]$ & $0.35[-8]$ & $0.35[-8]$ & $0.30[-13]$ \\
\hline
\end{tabular}




\section{FIGURES}

FIG. 1. Detachment of $\mathrm{H}^{-}$ion in bichromatic field with the frequencies $\omega=0.0043$ and $3 \omega$ and intensities $I_{1}=10^{10} \mathrm{~W} / \mathrm{cm}^{2}$ and $I_{2}=10^{9} \mathrm{~W} / \mathrm{cm}^{2}$ respectively. Differential detachment rate (in units $10^{-8}$ a.u.) as a function of the electron emission angle $\theta$ is shown for the first ATD peak (corresponding to absorption of $n=8$ photons of frequency $\omega$ ) and various values of the field phase difference $\varphi$ as indicated in the plots. Open symbols show results of calculations by Telnov et al [11] (in the $\varphi= \pm \frac{1}{2} \pi$ plot the open circles show the results for $\varphi=\frac{1}{2} \pi$ and open triangles these for $\varphi=-\frac{1}{2} \pi$ ). Solid curves show results of the present adiabatic theory (which coincide for $\varphi=\frac{1}{2} \pi$ and $\varphi=-\frac{1}{2} \pi$ as discussed in the text).

FIG. 2. Same as in Fig.1, but for the second ATD peak (corresponding to absorption of $n=9$ photons of frequency $\omega)$.

FIG. 3. Same as in Fig.1, but for the third ATD peak (corresponding to absorption of $n=10$ photons of frequency $\omega)$.

FIG. 4. Same as in Fig.1, but for intensities $I_{1}=10^{10} \mathrm{~W} / \mathrm{cm}^{2}$ and $I_{2}=10^{8} \mathrm{~W} / \mathrm{cm}^{2}$; the differential detachment rate is shown for the first ATD peak (corresponding to absorption of $n=8$ photons of frequency $\omega)$.

FIG. 5. Same as in Fig.4, but for the second ATD peak (corresponding to absorption of $n=9$ photons of frequency $\omega)$.

FIG. 6. Same as in Fig.4, but for the third ATD peak (corresponding to absorption of $n=10$ photons of frequency $\omega)$. 

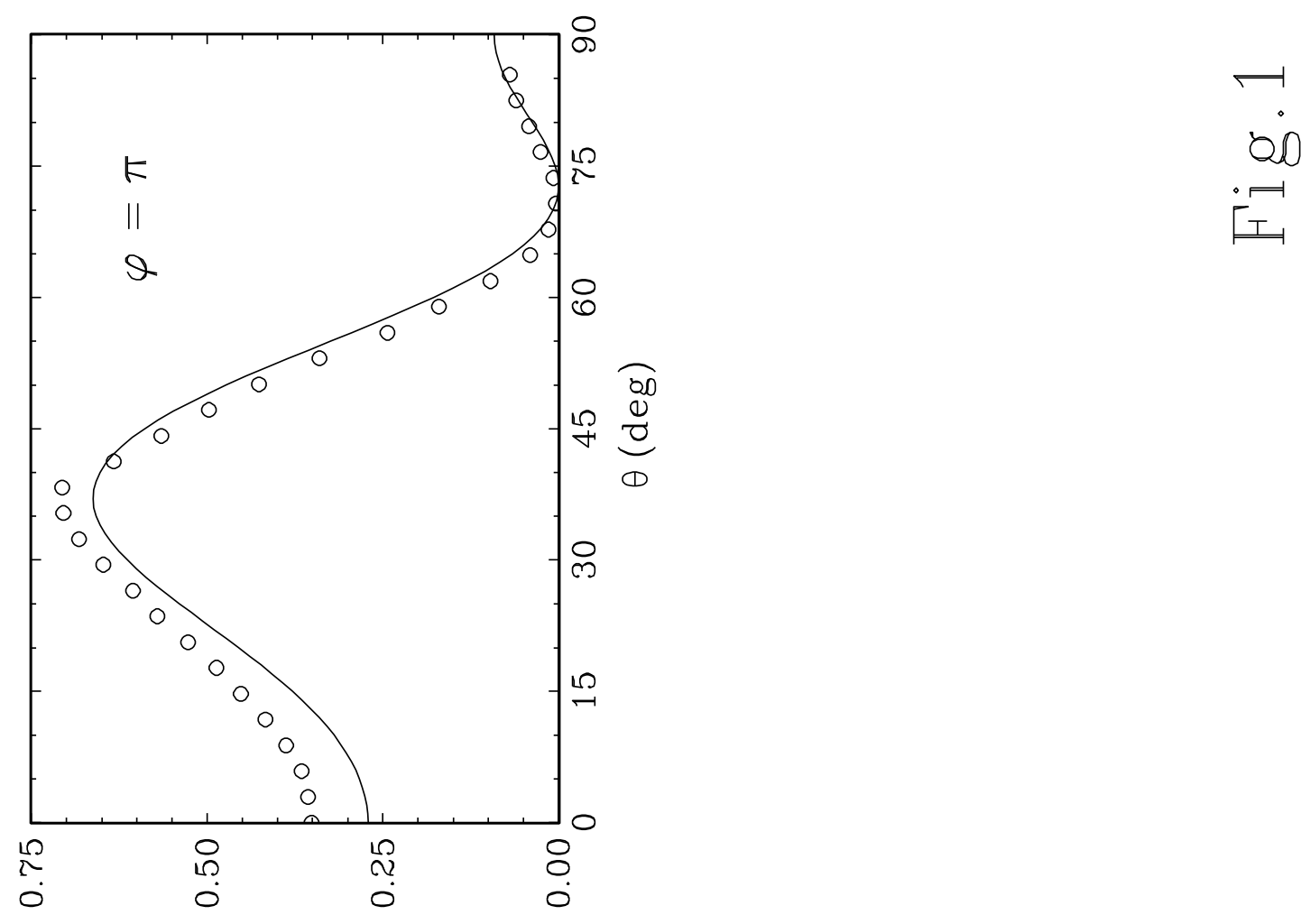

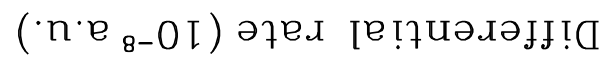

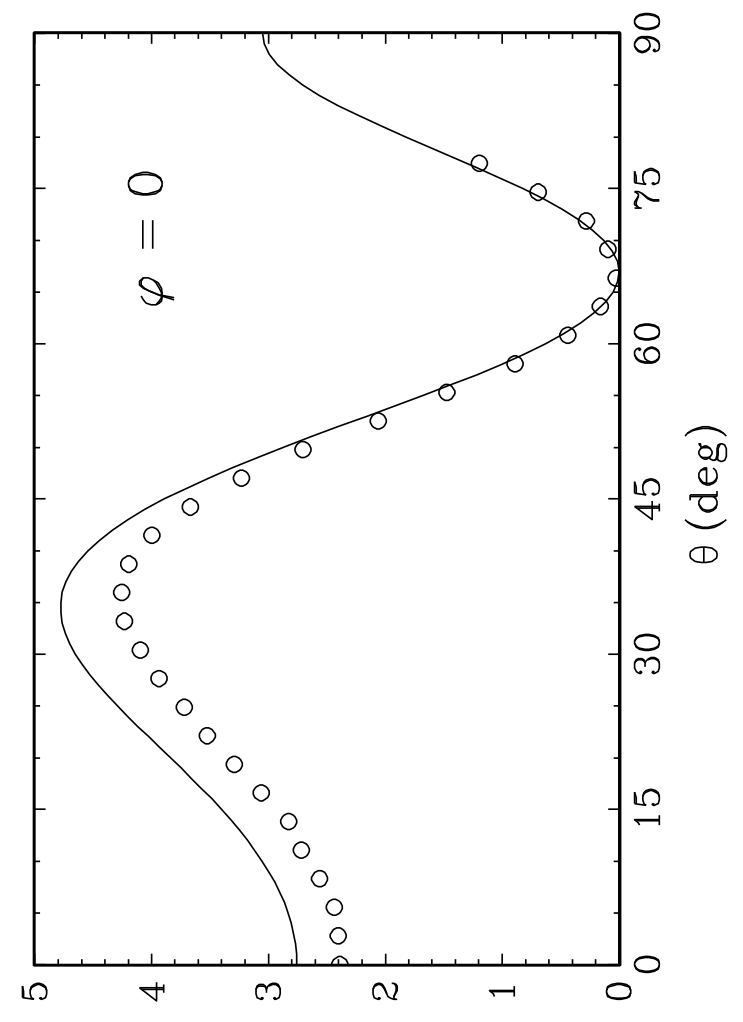

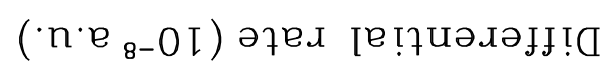

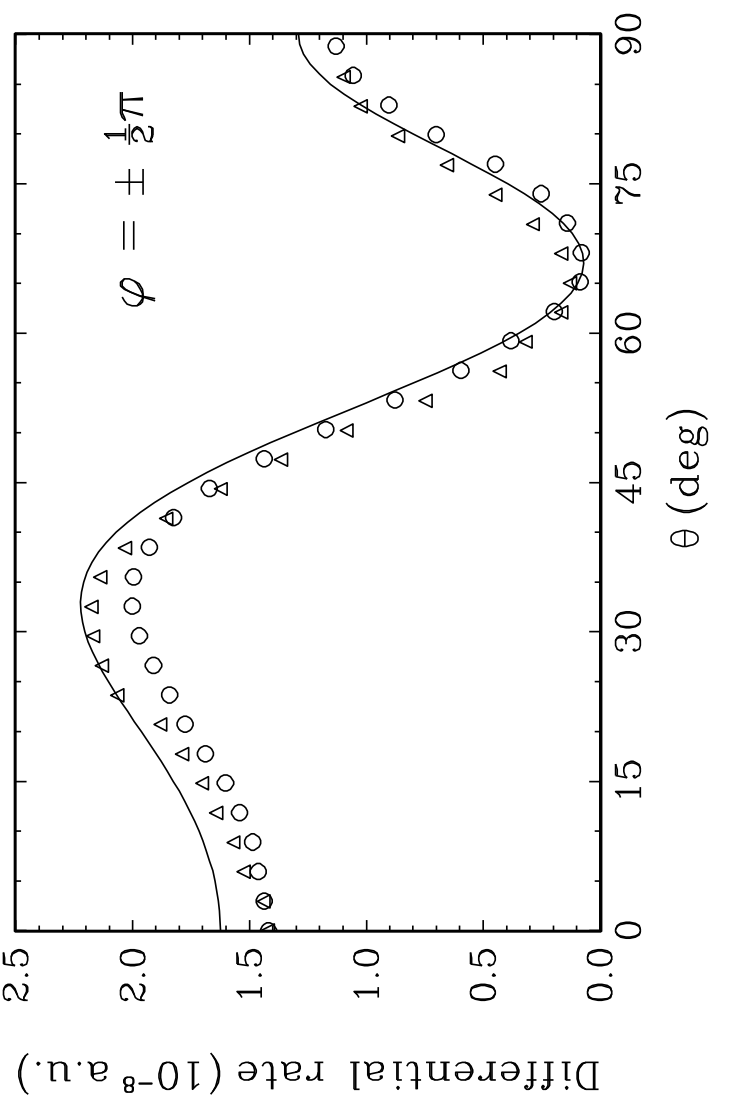




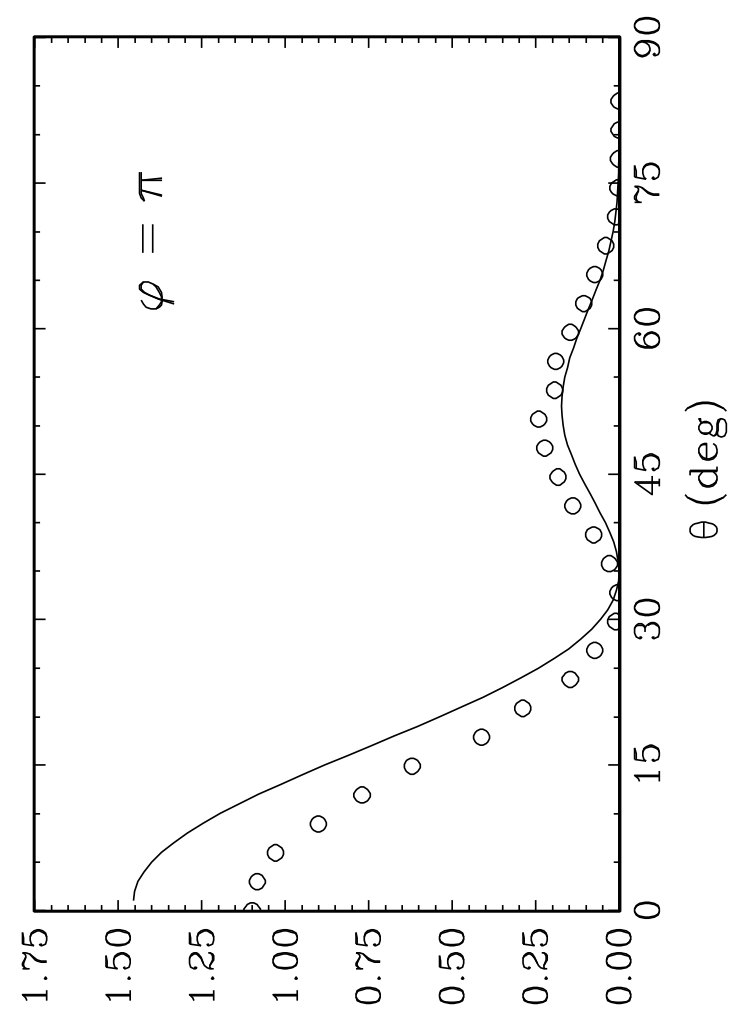

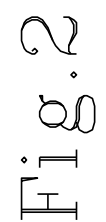

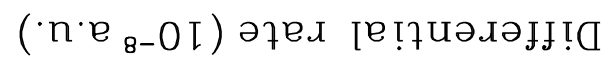
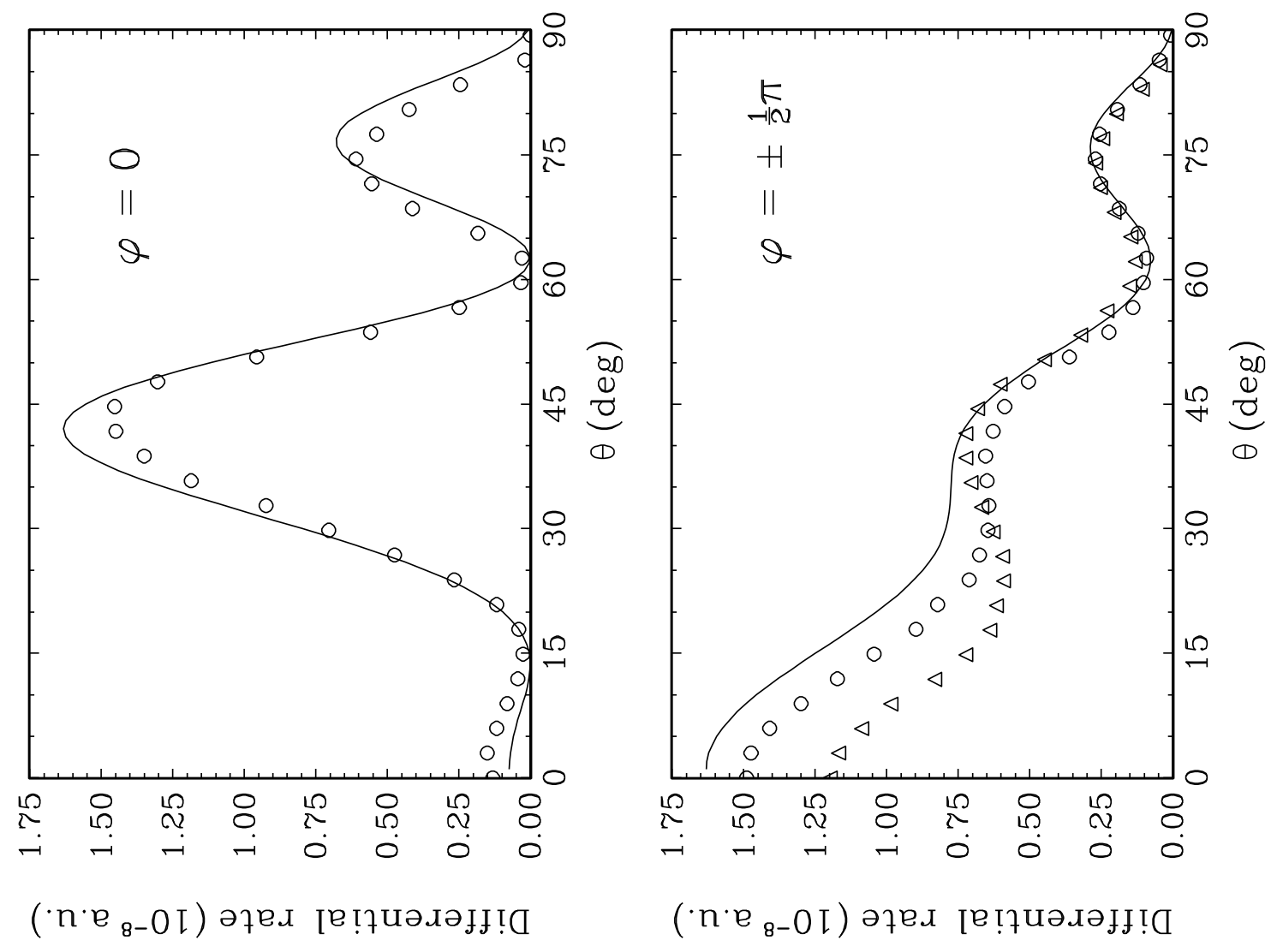

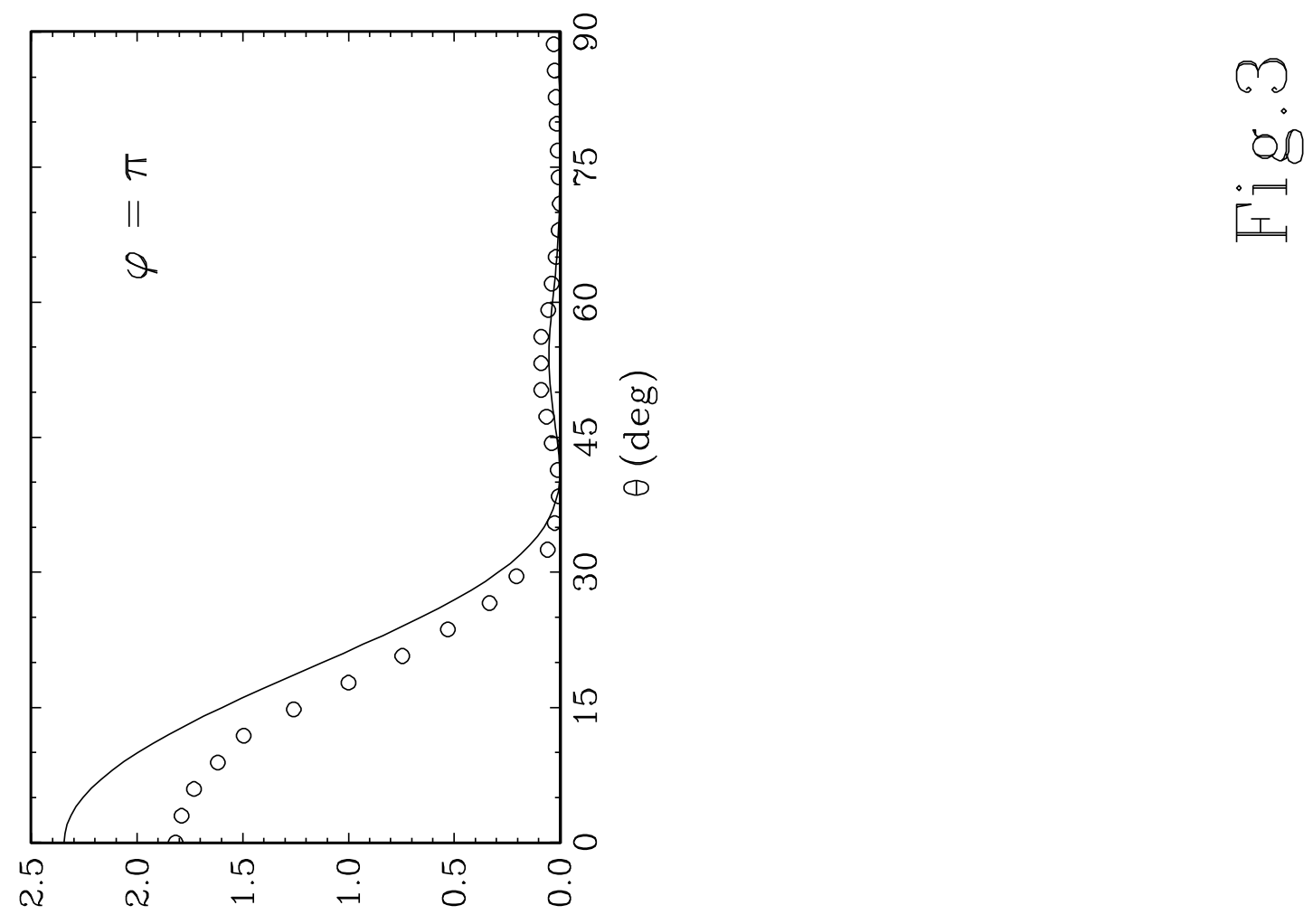

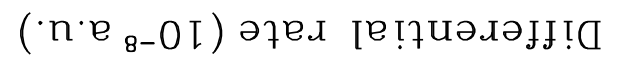
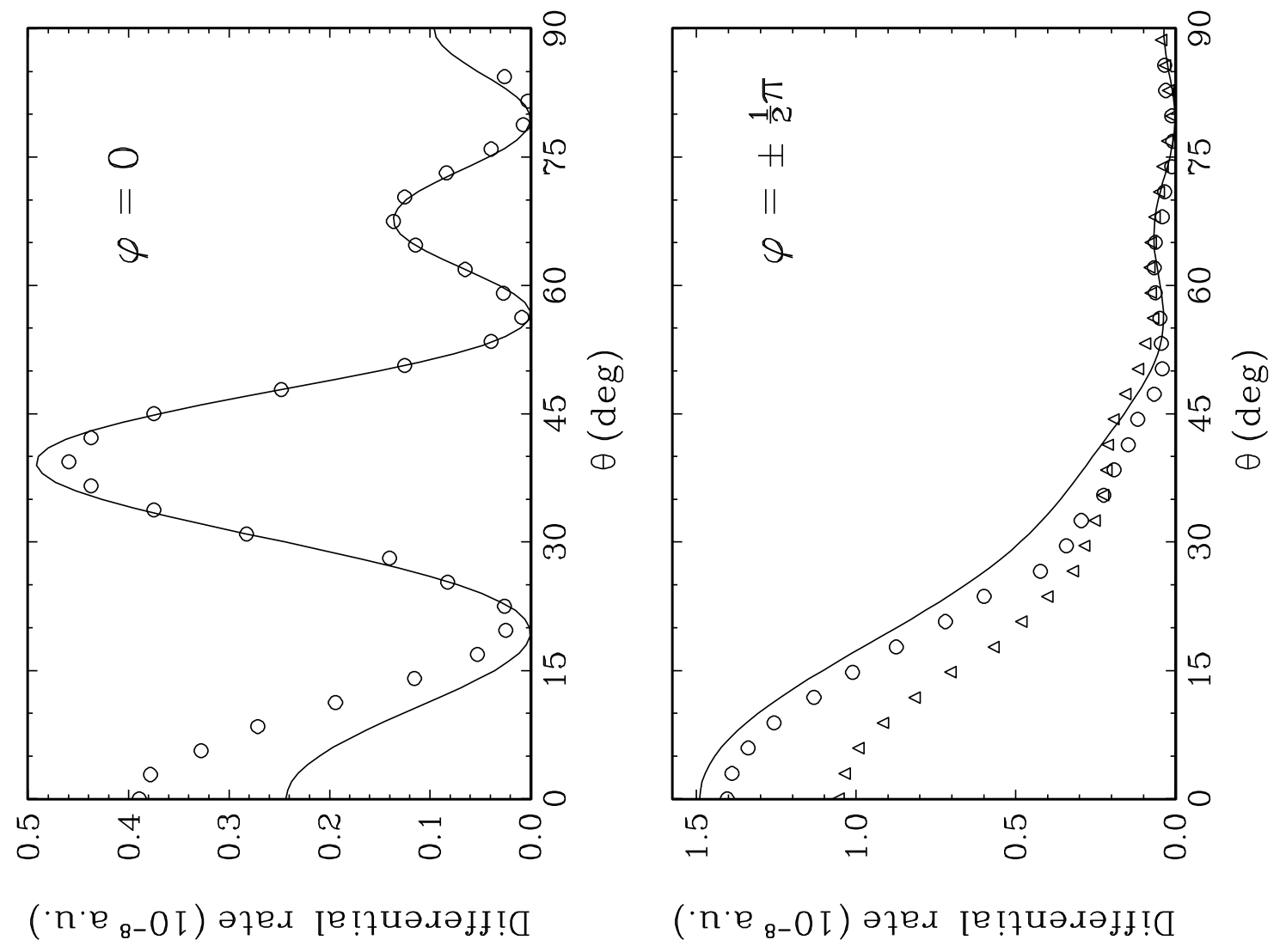

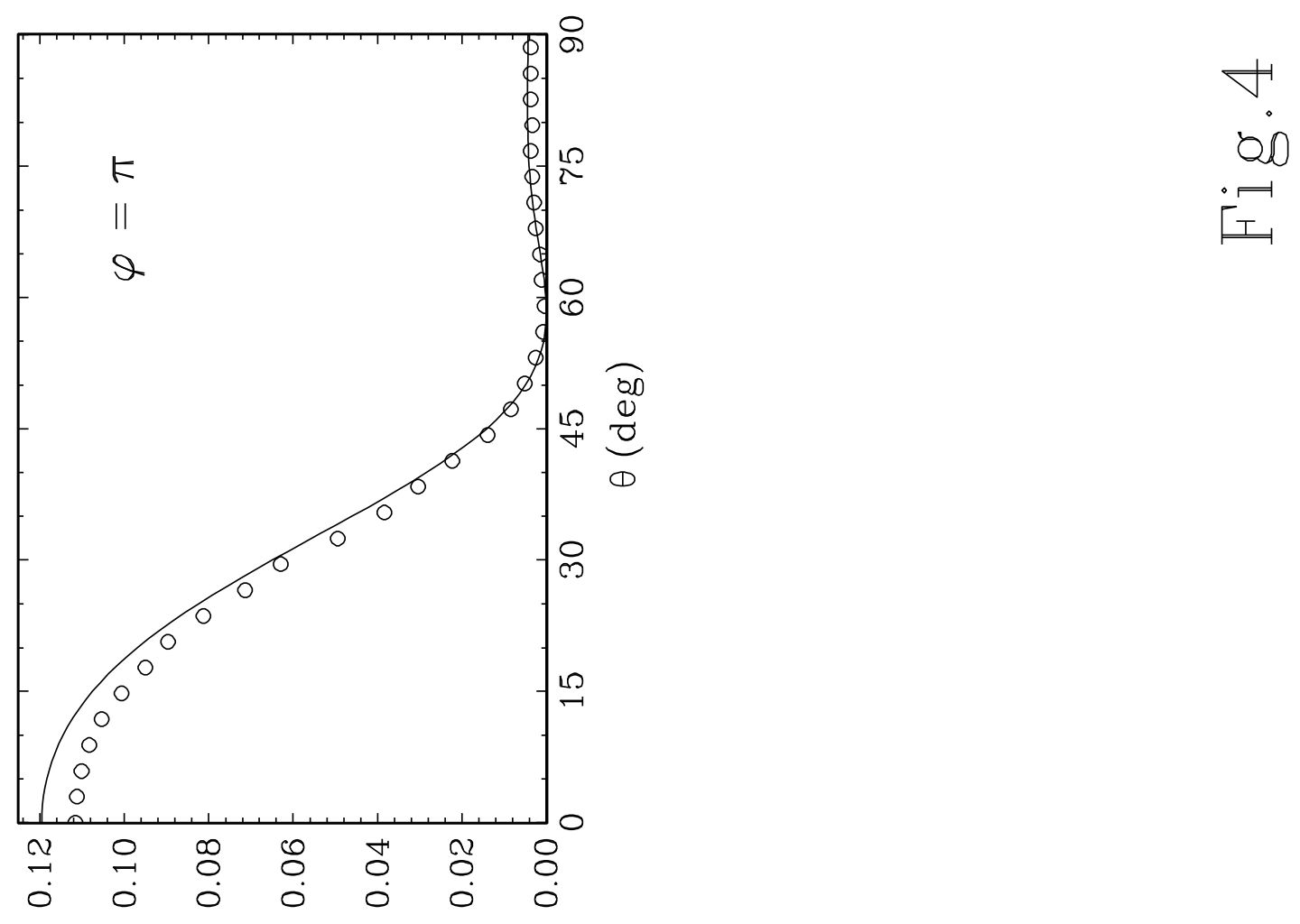

$\left(\cdot n \cdot e^{8-0 \tau}\right)$ әтел те тұนวләлт!
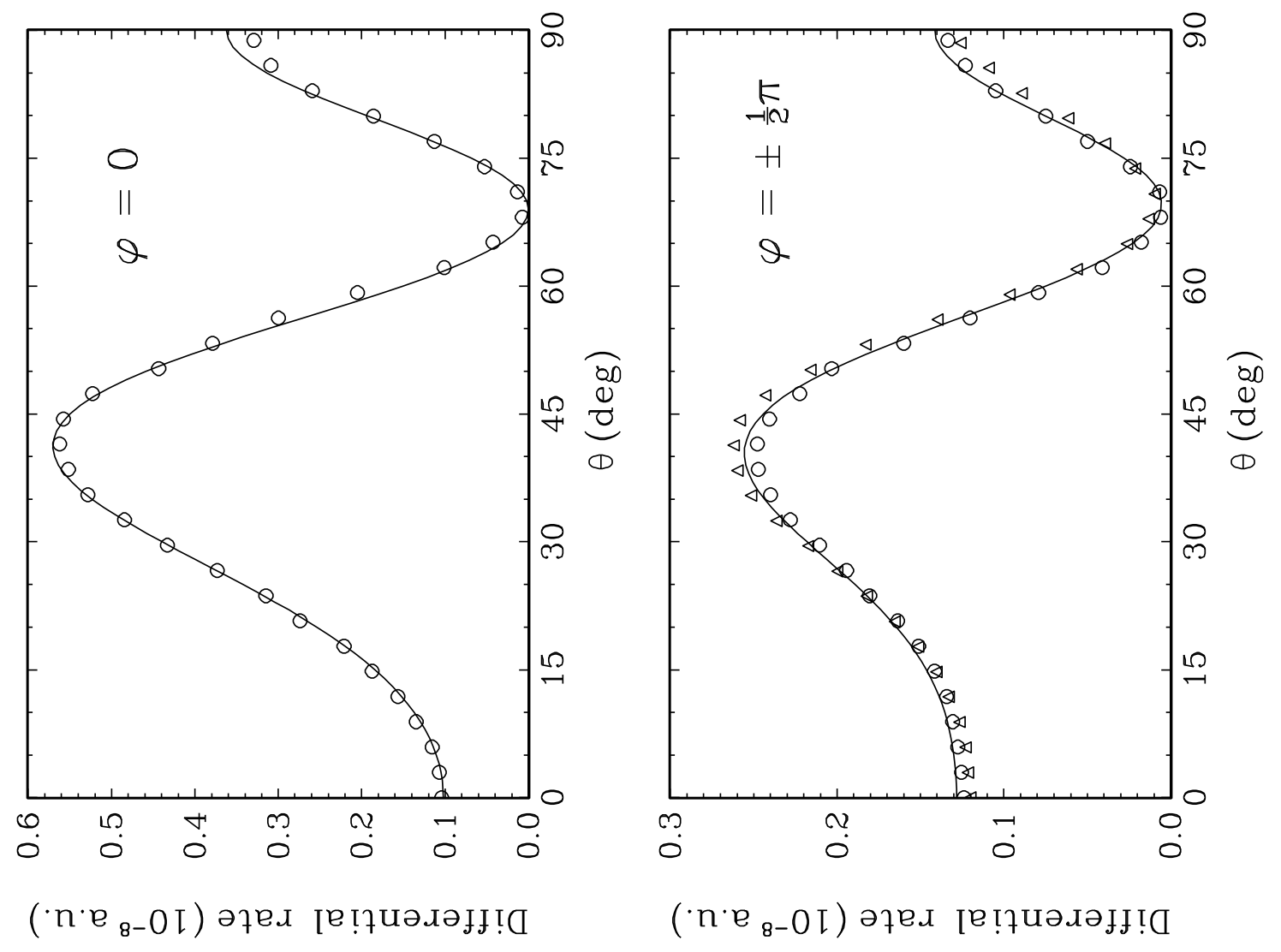

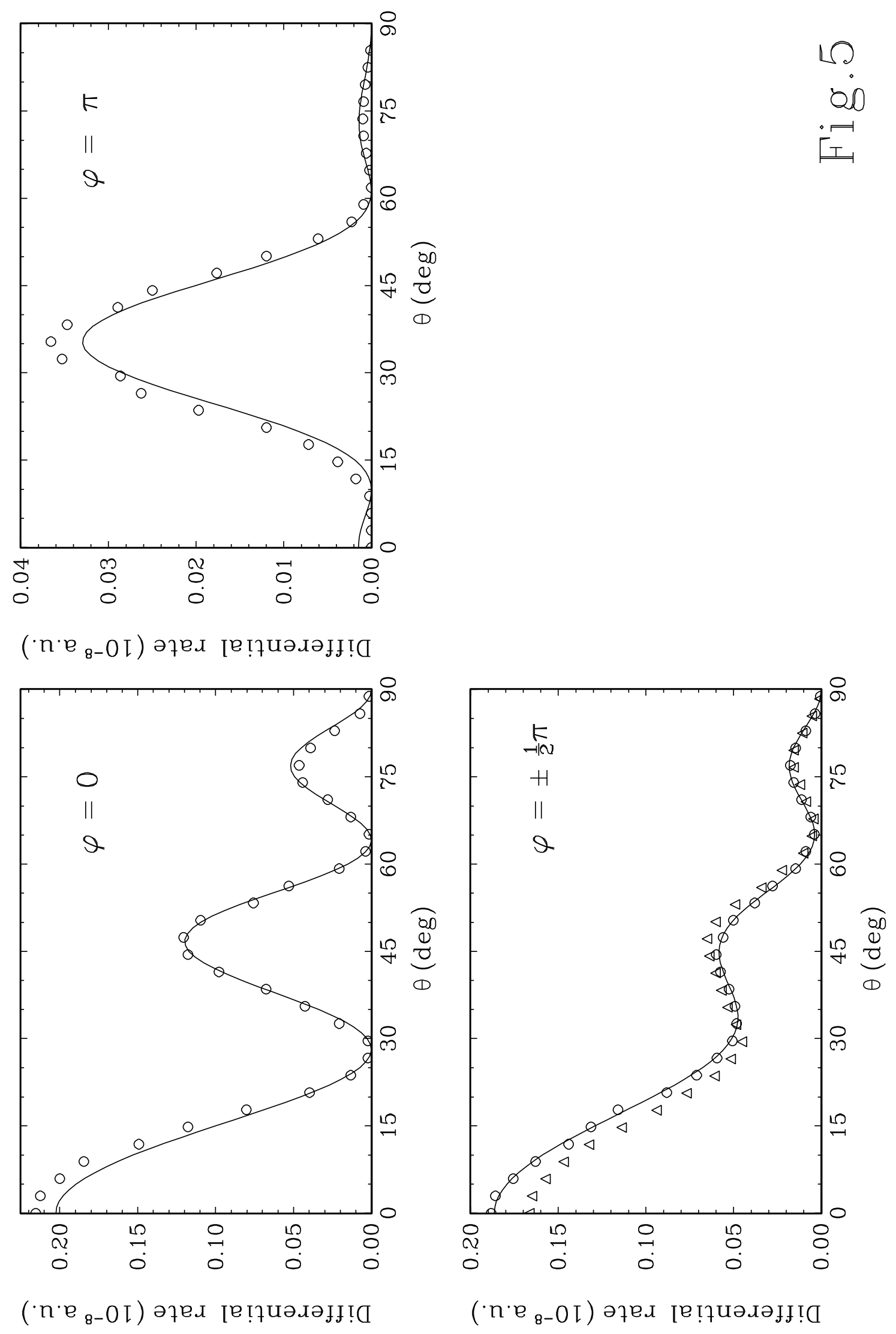

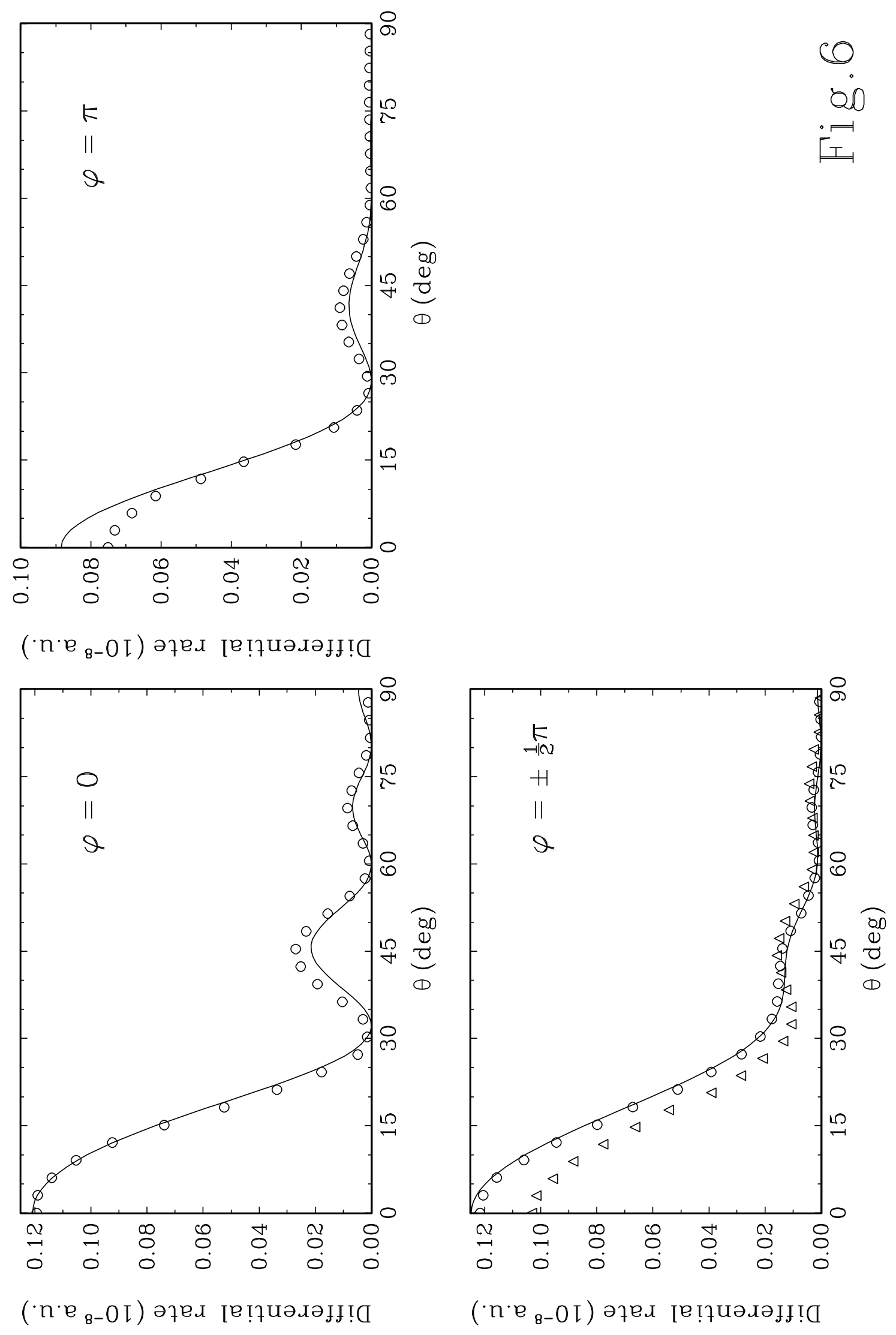